\title{
Multi-path selection in RPL based on replication and elimination
}

\author{
Tomas Lagos Jenschke, Remous-Aris Koutsiamanis, \\ Georgios Z. Papadopoulos, and Nicolas Montavont \\ IMT Atlantique, Irisa, UBL, France \\ \{firstname. lastname\}@imt-atlantique.fr
}

\begin{abstract}
IPv6 Routing Protocol for Low-Power and Lossy Networks $(\mathrm{RPL})$ is a distance vector routing protocol especially designed for the Internet of Things (IoT). RPL uses broadcast DODAG Information Object (DIO) messages to build a Destination Oriented Directed Acyclic Graph (DODAG) toward a root. Each node selects a parent node toward the root using a common Objective Function (OF). However, the use of a single route can affect the network reliability and the end-to-end latency. In this study, we propose to employ the Packet Replication and Elimination (PRE) principles to use parallel paths toward the DODAG root, over the IEEE 802.15.4 Time-Slotted Channel Hopping (TSCH) as a medium access. To this aim, we propose number of algorithms to select the second or the alternative parent in RPL. Furthermore, we study the advantages of using overhearing feature over correlated paths. Our simulation campaign conducted over Cooja, the simulator of Contiki OS, demonstrate that the use of overhearing in conjunction with PRE in RPL considerably improves the robustness of a wireless network by providing greater opportunity to a packet to reach its destination.
\end{abstract}

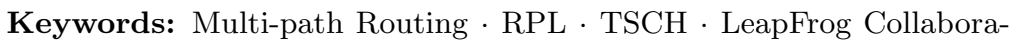
tion.

\section{Introduction}

The IoT are set of standards and technologies developed to connect embedded and smart objects to the Internet. Its low cost, environment adaptability and easy development have allowed the massive integration of these technologies in different application domains, such as the smart grid, Intelligent Transport System (ITS) or the Industry 4.0. The use of the IoT in these various applications however requires different quality of service, which is not an inherent features provided by IoT technologies. For example, the Industry 4.0 is especially concerned with the deterministic nature of the network, i.e., to rely on bounded delay and very high delivery ratio.

Many standard solutions have appeared during the last decade for wireless technologies for embedded devices. IEEE 802.15.4 [5] is a standard for low energy power devices focused on the industry. This standard is historically based on 
Time Division Multiple Access (TDMA) and Frequency Division Multiple Access (FDMA). Few years ago, the concept called TSCH [1] has emerged, which reduces the Multi-path fading by using frequency hopping. For extended networks where a destination node is out of the radio range of the source node, a routing protocol might be used on top of the Layer 2 technology. RPL is one of the most popular routing protocol for the IoT and is a distance vector routing protocol, which distributes the nodes within a tree hierarchy called DODAG. By default, each node has an option (route) to transmit a packet to a preferred node until it reaches the target. However is has been shown that reliability performance can be mitigated in real environment, and the retransmission schemes employed when a packet is loss introduce significant delay and jitter. In our previous works, we introduced Leapfrog Collaboration (LFC) [8], [4] which allows nodes to select additional parents to create alternate paths. To do so, we used the PRE concept, in which several copies of a data packet are introduced in a network to increase the probability of reception at the root node. In this paper, we further investigate LFC and study the effect of the parent selection algorithm. We introduce five algorithms for a node to select alternate parents, and compare their performance in the Cooja simulator.

The document is organized into five sections as follows. In Section 2 the RPL protocol, TSCH technique and the PRE mechanism are briefly explained. In Section 3, the different techniques proposed for the Alternative Parent (AP) selection to be evaluated are explained. In Section 4 contains the results obtained from the different methods proposed in Section 3. In Section 5, we find the different works carried out on multi-path in RPL before our work. Finally, Section 6 concludes our paper.

\section{Background}

\subsection{IEEE802.15.4}

TSCH is a recent channel access technique developed for IEEE 802.15.4. Its main objective is to reduce the impact of wireless fading on a network transmission. TSCH is composed of many timeslots that, in turn, are grouped into slotframes. In order to make this work, TSCH schedules a node to do what it has to do in a timeslot. The formula that uses TSCH to do its channel hopping is represented in the Equation 1.

$$
f=F(A S N+c h O F) \bmod n_{c h}
$$

Where $A S N$ is the slot offset, chOF is the channel offset, $F$ is the channel input and $f$ is the resultant frequency. TSCH is focused exclusively on the MAC layer, allowing the adjustment of time intervals in a wide range of protocols enabled for IPv6. IEEE802.15.4e currently defines the mechanisms necessary to carry out a communication through TSCH but does not establish the construction and maintenance policies of this, so there is a free interpretation. 


\subsection{RPL}

RPL is a distance vector routing protocol designed and developed by the IETF working group ROLL [12]. This protocol organizes the nodes in a DODAG to allow upward traffic from leafs to sensors, and propose different options for the downward routes. To build a DODAG, a DODAG root sends multicast DIO packets with the necessary information so that a node can join the network. A unicast DODAG Advertisement Object (DAO) message is sent upward to propagate destination information and construct downward routes. Within a RPL instance, there is an OF which defines how to combine different metrics to perform parent selection, i.e. choose which neighbor will be the default relay node toward the root. By default, a node has a single Preferred Parent (PP) selected from its parent list. Different metrics can be used:

- Hop Counting (HC): This metric consists of the minimum amount of hops necessary for the message to reach its destination. In contiki OS [7], the OF0 is designed to find the nearest root. i.e, the minimum number of relay nodes.

- Expected Transmission Count (ETX): ETX consists of a link quality metric, based on the average number of retransmissions needed to reach a given neighbor. This does not mean that it will be the shortest route, but it ensures the quality of the transmission links. To reach this objective, the OF uses the following formula defined by [10]:

$$
E T X_{i j}=\frac{\left|N_{t x_{d a t a}}\right|}{\left|N_{r x_{d a t a}}\right|} \times \frac{\left|N_{t x_{A C K}}\right|}{\left|N_{r x_{A C K}}\right|}=\frac{\left|N_{t x_{d a t a}}\right|}{\left|N_{r x_{A C K}}\right|}
$$

Where $N_{t x_{\text {data }}}$ is the number of transmitted messages, $N_{r x_{\text {data }}}$ is the number of received messages, $N_{t x_{A C K}}$ is the number of transmitted ACKs and $N_{r x_{\text {data }}}$ is the number of received ACKs.

\section{$2.3 \quad 6 \mathrm{TiSCH}$}

The IETF working group 6TiSCH [11] is in charge of the interworking between the IEEE802.15.4 TSCH and the IETF upper stack 6LoWPAN, RPL and CoAP. To make this possible, they are implementing an extra layer over TSCH MAC layer to adapt the scheduling technique to the specificities and requirements of the the upper layers. As is explained in [11] LLN needs to:

1. Provide a mechanism for two devices to negotiate the allocation and deallocation of cells between them.

2. Provide a mechanism for the device to monitor and manage the capabilities of a node several hops away.

3. Define a mechanism for these different scheduling mechanisms to coexist in the same network.

In this paper we assume a centralized scheduling where controller establishes the transmission and reception slots for all RPL nodes. 


\subsection{PRE}

We previously proposed LFC which increases the reliability and the determinism feature of a multi-hop network by using PRE concept. Replication means that several copies of a single packet are generated in the network (either by the source, by intermediate nodes, or both), and Elimination means that each node that receives a duplicates will discard it. In RPL, by default, a node can have one preferred route identified by its PP. To do the replication, LFC adds the use of an alternative route by choosing an AP from its parent set except for its chosen PP.

Once a node has chosen two PPs, it duplicates a packet to both its parents, and then send two copies of the same data packets. Note that the two parents may benefit from overhearing the transmission to the other parent, ever increasing the probability of receiving the packet. A node may select its PP in many different ways, and in the next sections we present five possible algorithms.

\section{Algorithms for multiple parent selection}

By default, RPL performs routing by forwarding packets via the selected PP. The selection of a PP amongst multiple candidate parents is made through its OF. This selection consists mainly of the ranking hierarchy, which increases as the DODAG tree becomes deeper. This type of selection avoids communication loops because a node can be a possible PP, if and only if, its rank is lower than that of its children. To find the most suitable route, there are different types of selection metrics, of which we chose to use ETX.

In this section we define different types of AP selection for Multi-path transmission. Note that if none of the potential parents of a node matches the selection process, or if several nodes do, then the one with the second best ETX will be chosen. It is also possible that no AP is selected in the case where a node has only one parent.

\section{$3.1 \quad$ Overhearing}

To take advantage of the nature of the wireless medium, nodes close to a transmission can overhear a packet. We implemented this feature, that we call overhearing, to let a potential parent receive a packet sent from a child to another parent. This allows a second opportunity for the information to reach its destination.

Within this work, the use of overhearing was incorporated into LFC, i.e., in all cases of Multi-path selection. In addition, we developed another algorithm where we only add the Overhearing to RPL (i.e., RPLO) without doing any duplication. 


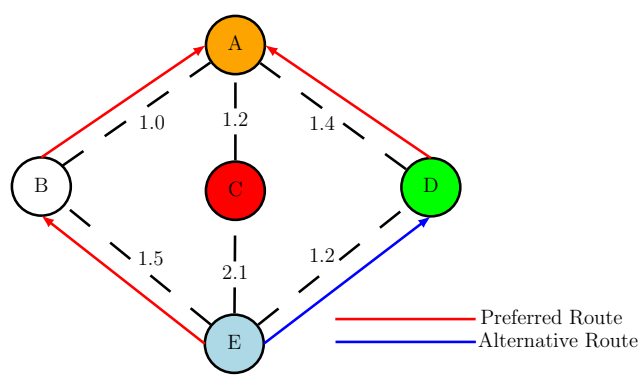

(a)

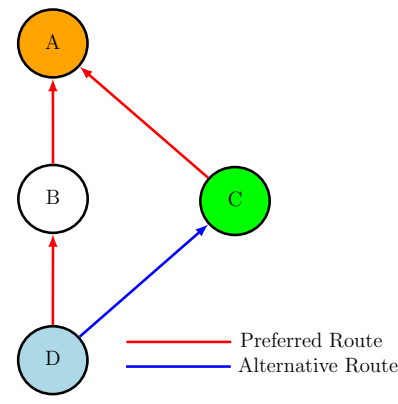

(b)

Fig. 1: (a) 2ETX illustration (b) CA illustration

\section{$3.2 \quad$ Second Best ETX (2ETX)}

This algorithm consists of obtaining an AP by selecting the node that has a lower ranking than the current node and that has the best link quality among the set of possible parents. In Figure (1a) a selection example is shown by the use of 2ETX where if we define the rankings such as R and link quality as M:

- $A_{R}<B_{R}$ and $A_{R}<C_{R}$ and $A_{R}<D_{R}$

- $E_{R}>B_{R}$ and $E_{R}>C_{R}$ and $E_{R}>D_{R}$

$$
\begin{aligned}
& E_{M}+B_{M}+A_{M}=2.5_{E T X} \\
& E_{M}+C_{M}+A_{M}=3.1_{E T X} \\
& E_{M}+D_{M}+A_{M}=2.6_{E T X}
\end{aligned}
$$

Since $2.5_{E T X}$ is the lowest value, node $\mathrm{B}$ will be the PP of node $\mathrm{E}$ and node $\mathrm{D}$ will be the AP of node $\mathrm{E}$ because $2.6_{E T X}<3.1_{E T X}$.

\subsection{Common Ancestor (CA)}

A node will select a node as its AP if it has a common ancestor with the PP. It means that the AP and the PP should have a common PP in their Metric Container (MC) [3]. If multiple potential parents exhibit the same characteristic, the one with the best ETX will be selected. Minet et al. in [6] call this algorithm the "Triangle Pattern". They also present the "Braided patter" which works similarly to the triangle pattern with the main difference being that the AP also has the chance to have an AP too. In our previous work [8], we used this algorithm.

Figure (1b) shows an example of CA where node $\mathrm{C}$ is the $\mathrm{AP}$ of node $\mathrm{D}$ and $\mathrm{A}$ is the common ancestor (potential parent) of node $\mathrm{D}$ and $\mathrm{C}$. In order to implement this algorithm, a node should advertise its list of potential parents to its children. The IETF draft [3] provides a proposal and defines a "Type-LengthValue" (TLV) field within the "Node State and Attribute" (NSA) object type in the MC of DIO packets to carry this information. 


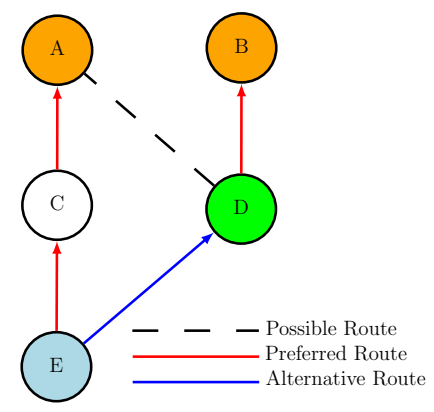

(a)

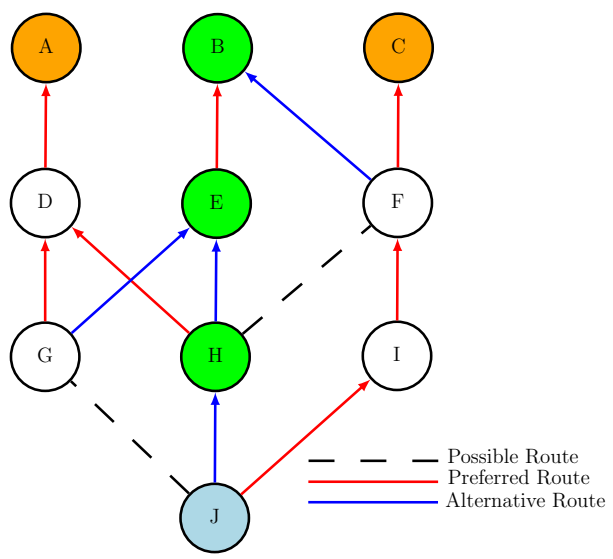

(b)

Fig. 2: (a) NCPA illustration (b) Disjoint illustration

\subsection{Non-Common Preferred Ancestor (NCPA)}

A node is selected as an AP if it has a different PP than the PP one. This selection mechanism works by storing in the DIO MC the PP address and the receiving child just has to compare the PP address of its potential parents. Figure (2a) illustrates the NCPA operation where node $\mathrm{A}$ is the preferred grandparent of node $\mathrm{E}$ and node $\mathrm{B}$ is the $\mathrm{PP}$ of node $\mathrm{D}$. Because $\mathrm{A}$ is different from $\mathrm{B}, \mathrm{D}$ will be the AP of node $\mathrm{E}$.

\subsection{Non-Common Ancestor (Disjoint)}

This algorithm consists in the selection of an AP if the current PP has a PP and AP different than the PP and AP from the potential node. In this way, the composition of a triangular pattern through an alternative node is deactivated, which allows the generation of one or several disjoint patterns according to the built network topology. Like the other selection techniques, the node will classify and select its potential AP ordered by the ETX metric. The Figure (2b) illustrates the operation of the Disjoint selection.

\section{Performance evaluation}

\subsection{Simulation environment}

In this study, we employed the COOJA network simulator for Contiki OS [7] to perform the performance evaluation campaign. To this aim, we defined three types of topology illustrated in Figure 3 and configured as shown in Table 1. 


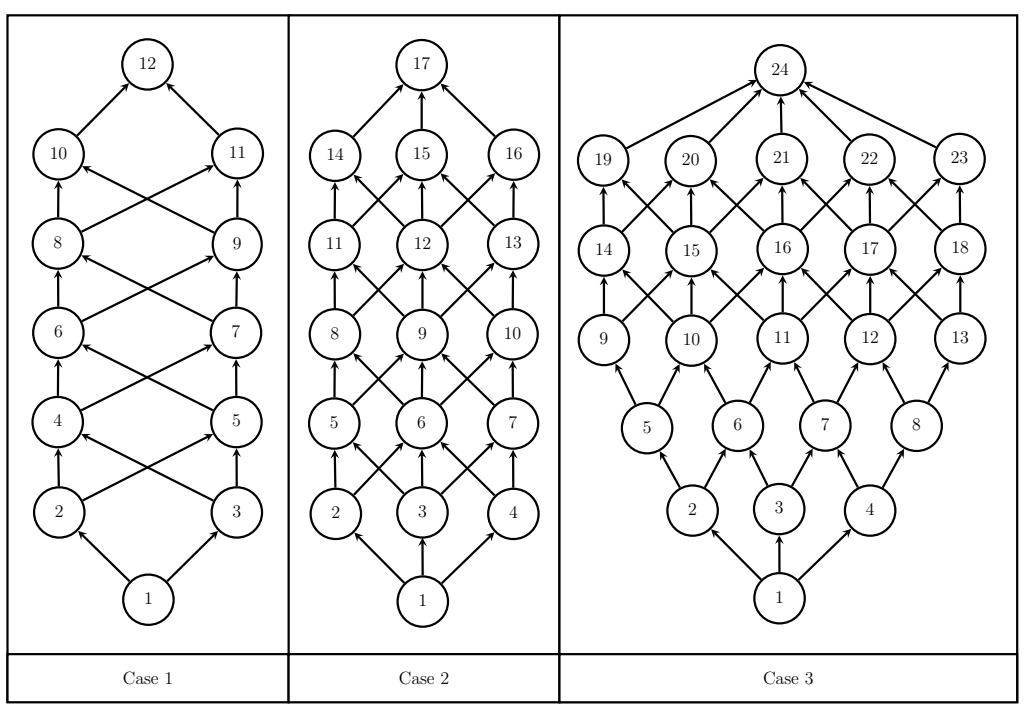

Fig. 3: Case 1: Topology with 12 nodes. Case 2: Topology with 17 nodes. Case 3: Topology with 24 nodes.

\begin{tabular}{|c|c|c|c|c|c|}
\hline & & \multicolumn{2}{|c|}{ Topology 1} & Topology 2 & Topology 3 \\
\hline Topology & & \multicolumn{2}{|l|}{ Multi-hop } & Multi-hop & Multi-hop \\
\hline \multicolumn{2}{|c|}{ Number of nodes } & 12 & & 17 & 24 \\
\hline \multicolumn{2}{|c|}{ SlotFrame length } & 53 & & 86 & 121 \\
\hline Duration & \multicolumn{2}{|c|}{ until 120 packets } & & period & $4 s e c$ \\
\hline Traffic & \multicolumn{2}{|c|}{$1 \mathrm{pkt} / 5 \mathrm{sec}$} & & eSlot length & $10 \mathrm{~ms}$ \\
\hline Routing & \multicolumn{2}{|l|}{ RPL } & \multicolumn{2}{|c|}{ Number of source } & 1 \\
\hline MAC & \multicolumn{2}{|c|}{$\mathrm{TSCH}$} & \multicolumn{2}{|c|}{ Number of channels } & 1 \\
\hline
\end{tabular}

Table 1: Simulation Setup.

- Topology 1 - simple use-case, where each node has two potential parents: 12 nodes, where leaf node 1 will be the transmitter and node 12 will be the DODAG root.

- Topology 2 - wider network, where each node has three potential parents: 17 nodes, where leaf node 1 will be the transmitter and node 17 will be the DODAG root.

- Topology 3 - dense network, where a node can have up to four siblings: 24 nodes, where leaf node 1 will be the transmitter and node 24 will be the DODAG root.

The MC implementation can be found in [2]. The simulation consists in the transmission of 120 packets every 5 seconds. We ran 10 trials for each algorithm and we tested six algorithms: default RPL, RPLO, 2ETX, CA, NCPA and Disjoint described in Section 3. 


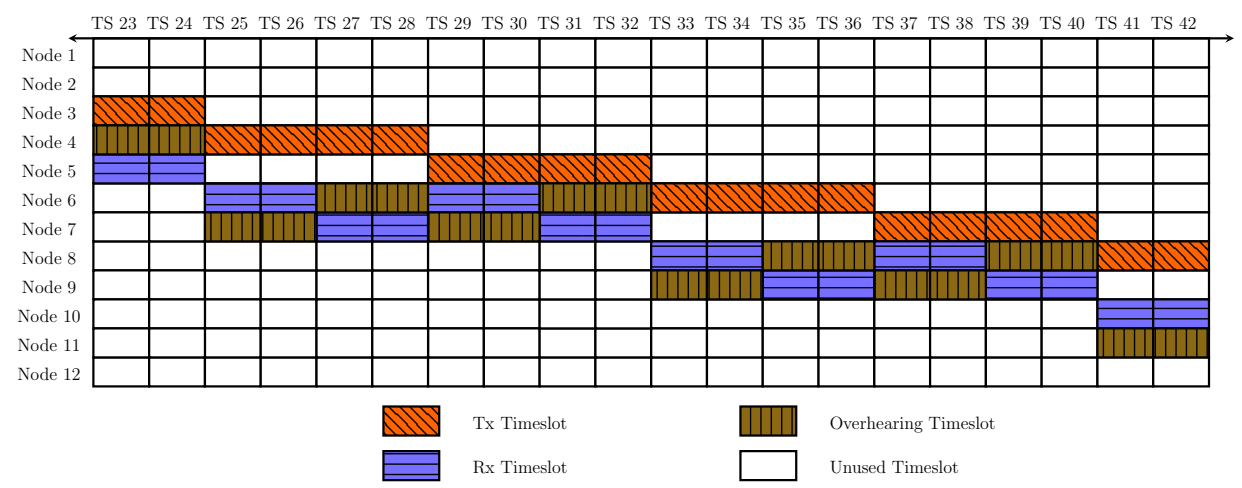

Fig. 4: Case 1 - TSCH Scheduling.

Figure 4 partially shows the configuration of the scheduling for Topology 1 . The transmission of a node to its possible parents follows a pattern from left to right. We configured two consecutive timeslots from a node to each upper node in the topology to allow the transmission of one data packet plus a retransmission if this first attempt failed. For some algorithms, we also defined overhearing timeslots, where a node will listen a transmission intended to another node. For example, in timeslot 33 , node 6 is sending to node 8 , and node 9 is also listening the transmission. The same pattern is also applied for Topology 2 and 3.

\subsection{Results}

Packet Delivery Ratio. We first measure the average Packet Delivery Ratio (PDR), i.e., the percentage of packets that arrive at the DODAG root. As shown in Figure 5, except for RPL, topologies 2 and 3 reach almost $100 \%$ PDR while in the case of Topology 1 they approach 95\% PDR. Stock RPL does not perform well with around $50 \%$ of PDR in all topologies. This highlights the improvement provided by the use of Multi-path and overhearing with respect to the standard implementation.

Delay and jitter. As shown in Figure 3, Topology 1 consists of 12 nodes where each of these has a set of two possible parents with the exception of the last hop. Considering that each timeslot lasts $10 \mathrm{~ms}$, the approximate delay can be calculated with the Equations in 4:

$$
\begin{array}{r}
\left(\left(\left(P_{2} \times N_{p s 2}\right)+\left(P_{3} \times N_{p s 3}\right)+1\right) \times T R\right)-T=\min _{\text {delay }} \\
\left(\left(P_{2} \times N_{p s 2}\right)+\left(P_{3} \times N_{p s 3}\right)+N_{s p}\right) \times T R=\max _{\text {delay }}
\end{array}
$$

where $P$ is the parent set, $N_{p s}$ are the nodes that have more than a single parent, $N_{s p}$ are the node that have only a single parent, $T$ is the timeslot duration and $R$ is the amount of transmissions plus retransmissions per attempt. 


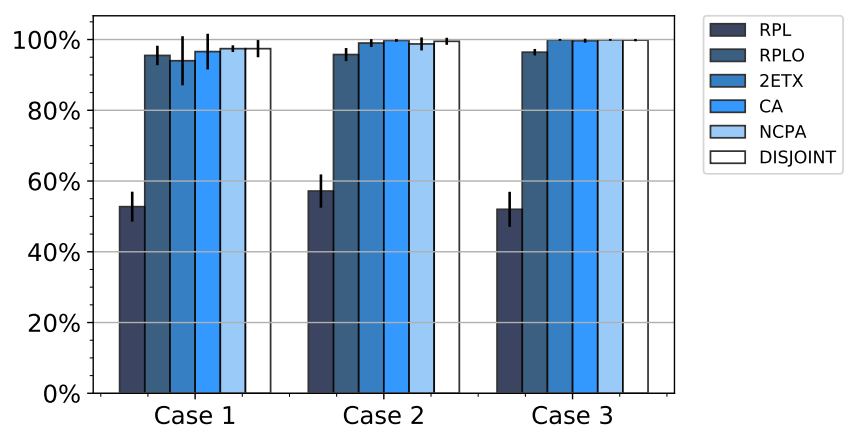

Fig. 5: Packet Delivery Ratio (PDR): percentage of received packets.

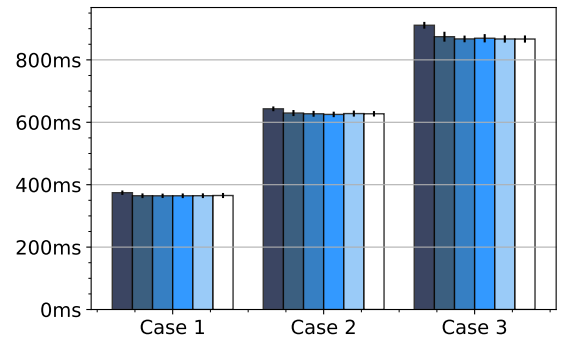

(a)

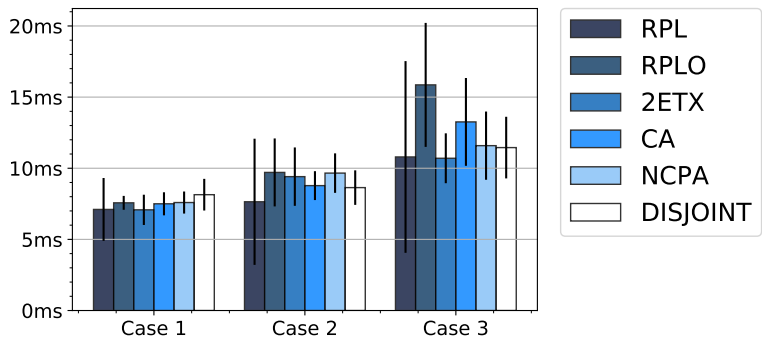

(b)

Fig. 6: (a) Packet delay: time for a packet to cross the network (b) Packet jitter: delay variation.

The minimum and maximum delay for the first, second and third topologies are shown in the Equations 5, 6 and 7, respectively.

$$
\begin{gathered}
(((2 \times 9)+1) \times 20)-10=370 \mathrm{~ms} \\
((2 \times 9)+2) \times 20=400 \mathrm{~ms} \\
(((2 \times 8)+(3 \times 5)+1) \times 20)-10=630 \mathrm{~ms} \\
((2 \times 8)+(3 \times 5)+3) \times 20=680 \mathrm{~ms} \\
(((2 \times 11)+(3 \times 7)+1) \times 20)-10=870 \mathrm{~ms} \\
((2 \times 11)+(3 \times 7)+5) \times 20=960 \mathrm{~ms}
\end{gathered}
$$

Figure (6a) shows the average delays obtained during the simulations where all tend to present minimum delay, while in Figure (6b) the average of the standard deviation (Jitter) between packets is shown.

As it can be observed that the algorithms have a similar pattern when their results are compared. The main difference is found in Figure (6b), Topology 3, where the jitter of the RPLO and CA algorithms are more important than the others. In RPLO, a node never duplicates packets, but several copies of each data 


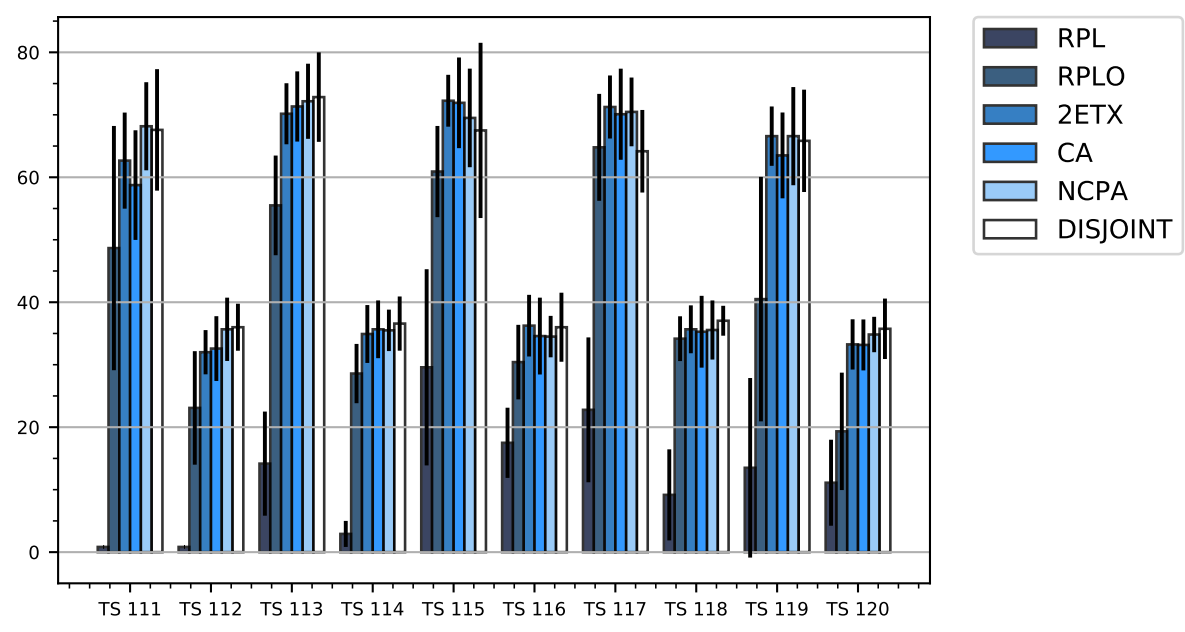

Fig. 7: Percentage of packets that follow a path.

packets are still created because there are multiple receivers for each transmission (due to the overhearing). This results in a more uniform distribution of the copies between all siblings at the last hop, as shown in Figure 7 . This figure reveals the reception traffic of the DODAG root from each of its 5 children in Topology 3 , where the $\mathrm{X}$ axis corresponds to timeslots for each transmission from the root's children to the root from left to right. For example, TS 111 and TS 112 correspond to transmission and retransmission of node 19, TS 113 and TS 114 of node 20 and so on. We observe two things: $i$ ) RPLO shows less traffic on each children ii) it is more distributed on each children, leading to higher jitter.

Figure 8 represents the percentage of packets received for the first time in each timeslot. Thus, it represents a CDF, i.e., the proportion of packets received in all possible timeslots. We can see that the distribution is concentrated in TS 111 or node 19 , which corresponds to a jitter $=0$. This phenomenon is due to the nature of the static scheduling. The traffic will behave like a priority queue, since, for each loss within a timeslot, the next attempt is made in the following timeslot. This explains the low jitter variance between the algorithms and the low delay for each of the transmissions.

Because default RPL does not have Multi-path and overhearing, its information receptions are not subject to TS 111 since it is unidirectional. In the case of RPLO and CA, their distribution is focused on the overhearing nodes because RPLO only follows the route of its PP and CA makes its way based on a triangular pattern. If we analyze Figure 8, we can observe a small variation of reception in TS 113 and TS 115 respectively, due to the distributive nature of these two algorithms.

In the case of 2ETX, this can be totally variable because its distribution focuses on the selection of the best link quality, while NCPA and Disjoint seek to expand as much as possible within the DODAG tree. This tends to happen across all the nodes and, in turn, to TS 111. 

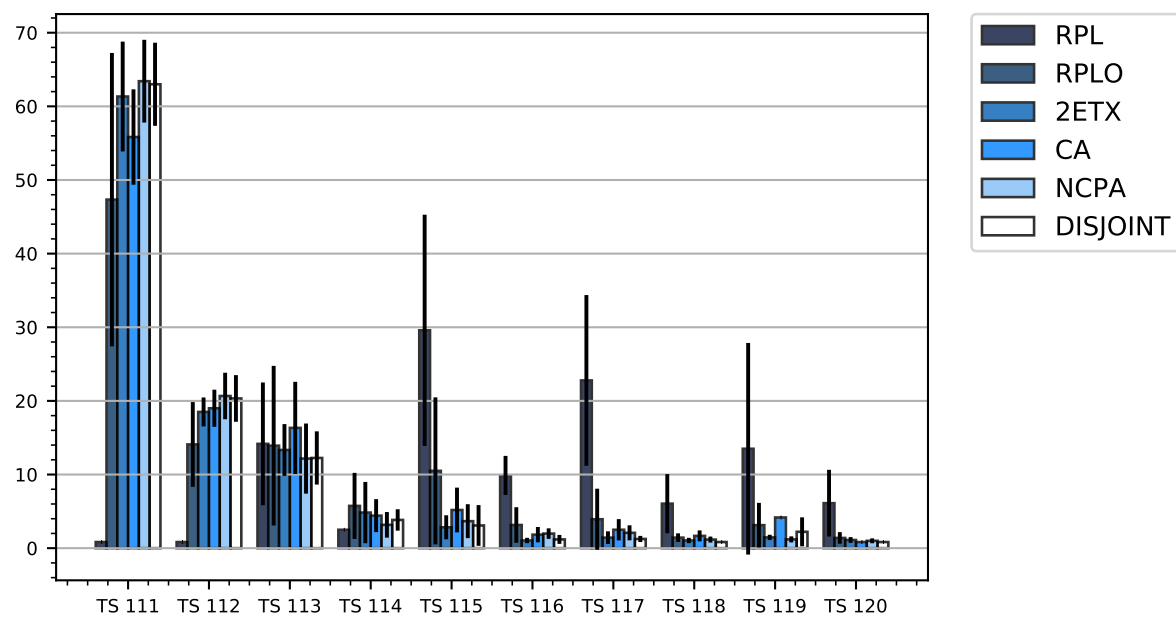

Fig. 8: Path of the first copy received by the DODAG root.

\section{Related Work}

A number of works related with multi-path in RPL have been conducted in the literature, focusing either on improving energy consumption or network reliability. However, to the best of our knowledge, there is no work that investigated the alternative parent and route selection for the Multi-path objective.

In [9], they propose the construction of a scheduling architecture to allow the transmission of multiple routes between a node and its different route options. To reach this goal, they adapt the transmit scheduling to resend the data packet to its different neighbors by choosing a randomly free slot and retrieving the used slots of their neighbors nodes. RPL is adapted also to take into account the delay and the packet delivery before a deadline. This is done with the purpose of handling the deadline transmission at each hop that the packet does.

In [6] the authors propose and analyze three different types of patterns. Each pattern is intended to increase the reliability of the communication by using an alternative route in addition to its preferred route. The result of each pattern is the duplication of packets in each node given more chances that the message reaches its destination. Consequently, the increase in reliability is proportional to the number of available paths that the node posses to transmit a packet. The drawbacks of the increase in possible paths is a higher networking overhead and a higher energy consumption.

\section{Conclusion}

In this work, we investigated the potentials of PRE scheme over parallel paths towards the DODAG root. To do this aim, we proposed four algorithms, the 
2ETX, CA, NCPA and Disjoint where overhearing function was employed, and we compared them against default RPL and RPLO.

Our performance simulation reveals that the PDR improves considerably when there is a means of replication in the medium. Regardless of whether RPLO does not have a AP, its performance is similar to the algorithms with replication. It was also observed that due to the dispersion of NCPA and Disjoint, the use of overhearing replicates the message to the nodes in their majority.

\section{Acknowledgements}

This work was partially performed and supported under the TPI ANR-17-CE100007-01 project of the French National Research Agency (ANR).

\section{References}

1. IEEE. IEEE Standard for Local and metropolitan area networks - Part 15.4: Low-Rate Wireless Personal Area Networks (LR-WPANs) Amendment 1: MAC sublayer. IEEE Std.802.15.4e-2012, April 2012.

2. A Koutsiamanis. The Contiki Operating System. https://github.com/ariskou/ contiki/tree/draft-koutsiamanis-roll-nsa-extension, July 2018.

3. R. Koutsiamanis and et al. RPL DAG Metric Container (MC) Node State and Attribute (NSA) object type extension. draft-koutsiamanis-roll-nsa-extension-01, IETF, roll, January 2018.

4. R. A. Koutsiamanis, G. Z. Papadopoulos, X. Fafoutis, J. M. Del Fiore, P. Thubert, and N. Montavont. From Best-Effort to Deterministic Packet Delivery for Wireless Industrial IoT Networks. IEEE Transactions on Industrial Informatics, 2018.

5. H. Kurunathan and et al. IEEE 802.15.4e in a Nutshell: Survey and Performance Evaluation. IEEE Communications Surveys \& Tutorials, February 2018.

6. P. Minet and et al. Increasing Reliability of a TSCH Network for the Industry 4.0. 16th IEEE International Symposium on Network Computing and Applications, November 2017.

7. Contiki OS. Contiki: The Open Source OS for the Internet of Things. http: //www. contiki-os.org, March 2018.

8. G. Z. Papadopoulos, T. Matsui, P. Thubert, G. Texier, T. Watteyne, and N. Montavont. Leapfrog Collaboration: Toward Determinism and Predictability in Industrial-IoT applications. In Proceedings of the IEEE International Conference on Communications (ICC), 2017.

9. B. Pavković and et al. Multipath opportunistic RPL routing over IEEE 802.15.4. International Conference on Modeling, Analysis and Simulation of Wireless and Mobile Systems, October 2011.

10. G Rajalingham and et al. Quality of Service Differentiation for Smart Grid Neighbor Area Networks through Multiple RPL Instances Gowdemy. Proceedings of the 10th ACM symposium on QoS and security for wireless and mobile networks Q2SWinet '14, September 2014.

11. T. Watteyne and et al. Using IEEE 802.15.4e Time-Slotted Channel Hopping (TSCH) in the Internet of Things (IoT): Problem Statement. IETF, RFC 7554 , October 2015.

12. T. Winter and et al. RPL: IPv6 Routing Protocol for Low-Power and Lossy Networks. IETF, RFC 6550, March 2012. 